\title{
Study on Imitating Grinding of Two-Dimensional Ultrasonic Vibration Turning System
}

\author{
Leping Liu*, Wen Zhao, and Yuan Ma \\ Key Laboratory of Ministry of Education for Conveyance and Equipment, \\ East China Jiaotong University, 330013 Nanchang, China \\ jxllp@163.com, wenwen0537@126.com, mayuan417@126.com
}

\begin{abstract}
According to turning instead of grinding process requirements and traditional working principle of ultrasonic vibration turning, a new idea of imitating grinding of two-dimensional ultrasonic vibration turning is brought firstly, and the mechanism of two-dimensional ultrasonic vibration turning is elaborated. Based on the MATLAB software, grinding trajectory simulation and honing trajectory simulation are carried out. Moreover, the design of ultrasonic transducer and ultrasonic horn is completed. Finally, the required mode shapes and resonance frequency are obtained by using the finite-element method, the results prove that the theoretical analysis is correct.
\end{abstract}

Keywords: Two-dimensional, Ultrasonic vibration, Turning; Grinding, Finite element.

\section{Introduction}

Ultrasonic vibration cutting appears in the early 20th century, because of good processing effect in difficult-to-machine materials and difficult processing procedures of common materials. It has aroused great concern to experts and scholars at home and abroad, and actively has carried out research and development. Currently, it has infiltrated into the automotive, aerospace, defense and other areas of the various processing [1].

The traditional ultrasonic vibration turning can reduce cutting force and cutting temperature. It also can improve the machining accuracy and surface quality. But in traditional ultrasonic vibration turning process, flank will rub and strike processed workpiece surface, when tool and workpiece are in the separated states, which seriously affectes the quality of the machined surface and tool life [2,3]. In order to solve the problem above, a new idea of imitating grinding of two-dimensional ultrasonic vibration turning is brought. This improves the quality of ultrasonic vibration turning and realizes turning instead of grinding.

\section{Traditional Ultrasonic Vibration Turning Mechanism}

The traditional ultrasonic vibration turning is to apply a certain frequency and amplitude of vibration to the tool, which improves the effect of turning [4].

* Corresponding author. Tel.: 13970086701. 
Tool's displacement in the cutting direction:

$$
x=a \sin (\omega t)
$$

Tool's vibration speed:

$$
v_{v}=a \omega \cos (\omega t)
$$

Tool's vibration acceleration:

$$
a_{t}=-a \omega^{2} \sin (\omega t)
$$

In formula (1) and formula (2), $a$ is vibration amplitude for the tool, $\omega$ is vibration angular frequency. Workpiece cutting speed is $v$. The tool separates from workpiece, when $v<a \omega$. The tool and the workpiece touch, when $v>a \omega$, so repeated cycles. For this reason, tip bears alternate pulling stress and compressive stress with the high-frequency ultrasonic vibration.

\section{Two-Dimensional Ultrasonic Vibration Turning Structure and Working Principle}

Ultrasonic processing system consists of ultrasonic generator, ultrasonic transducer, horn, tools, technology devices and so on. Ultrasonic transducer is the core of the system components.

Fig. 1 is a diagram of two-dimensional ultrasonic transducer. $A$ ' shows front shroud of transducer, $C^{\prime}$ shows back shroud of transducer, and $B^{\prime}$ shows piezoelectric ceramic stack.

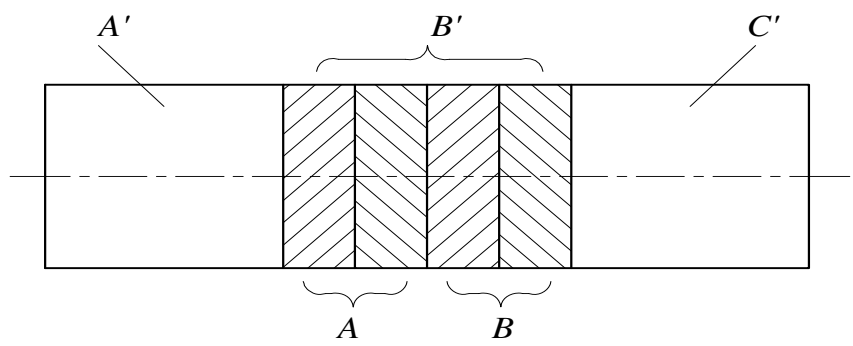

Fig. 1. The structure of two-dimensional ultrasonic transducer

Piezoelectric ceramic stack is composed of 4 piezoelectric ceramic disks, which is divided into group $A$ and group $B$. Each group is composed of 2 axial polarized ring piezoelectric ceramic disks. In group $B$, each of piezoelectric ceramic disks is splited into a half circle, and the two semi-circular is polarized in the opposite direction. Two piezoelectric ceramic disks are stacked in reverse polarity, and the arrangement is shown in Fig. 2. 


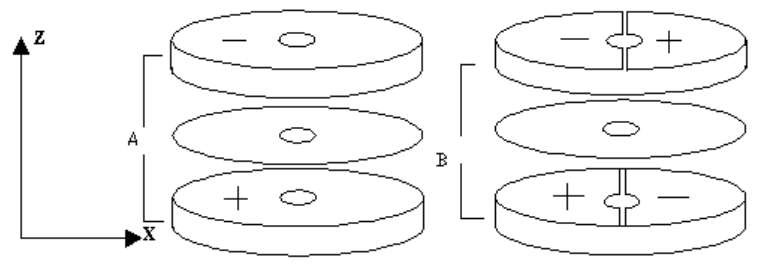

Fig. 2. The arrangement of piezoelectric ceramics

Adding AC electric field to group A, piezoelectric ceramic disks produce vibration from top to bottom (Z-axis). And adding AC electric field to group $B$, Piezoelectric ceramic disks produce bending vibration from left to right.

$\mathrm{X}$-axis and Z-axis vibration displacement:

$$
\begin{gathered}
x=a \sin (2 \pi f t) \\
z=b \sin (2 \pi f t+\varphi)
\end{gathered}
$$

In formula (4) and formula (5), $a$ is amplitude for the X-axis, $b$ is amplitude for the Z-axis, and $\varphi$ is phase difference between the two directions. According to formula (4) and formula (5), equation of motion can be written as follows:

$$
\frac{z^{2}}{b^{2} \sin ^{2} \varphi}+\frac{b^{2} x^{2}}{a^{2} b^{2} \sin ^{2} \varphi}-\frac{2 \cos \varphi}{a b \sin ^{2} \varphi} x z=1
$$

Required ultrasonic turning track can be obtained by adjusting the phase differernce of the AC electric field which is added to Piezoelectric ceramic disks.

\section{Two-Dimensional Ultrasonic Turning Simulates Grinding, Honing Track}

Grinding technology has extensive application in precision machining and ultra precision machining [5,6,7]. The movement of disseminated abrasive is at random. This paper mainly simulates the motion trace of sedentary abrasive. The trajectory of a single abrasive grit is shown by Fig. 3 .

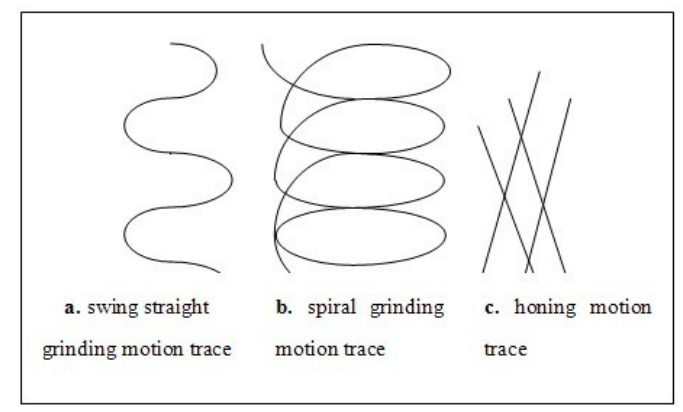

Fig. 3. Grinding, honing motion trace 
Tool vibration displacement on the X-axis is shown in formula (4). With the velocity $v$, tool along the Z-axis does uniform linear motion, which is also called the feed motion. Then simulative trajectory is gotten, as shown in Fig.4. This track is similar to swing straight grinding motion trace which is shown by Fig.3(a). The parameters are obtained as follows: $a=12 \mu \mathrm{m}, f=20 \mathrm{kHz}, \omega=2 \pi f=1.257 \times 10^{5} \mathrm{rad} / \mathrm{s}$, $v=20 \mathrm{~m} / \mathrm{min}$.

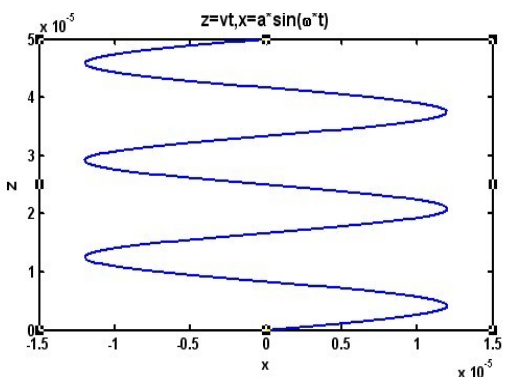

Fig. 4. Simulation trajectory

The AC electric field is added to group $A$ and group $B$ at the same time, and the phase difference of electric field is $\varphi=\pi / 2$. According to formula (4) and formula (5), the simulative trajectory is shown in Fig.5. The parameters are obtained as follows: $a=12 \mu \mathrm{m}, b=16 \mu \mathrm{m}, f=20 \mathrm{kHz}, \omega=2 \pi f=1.257 \times 10^{5} \mathrm{rad} / \mathrm{s}$, $v=20 \mathrm{~m} / \mathrm{min}$.

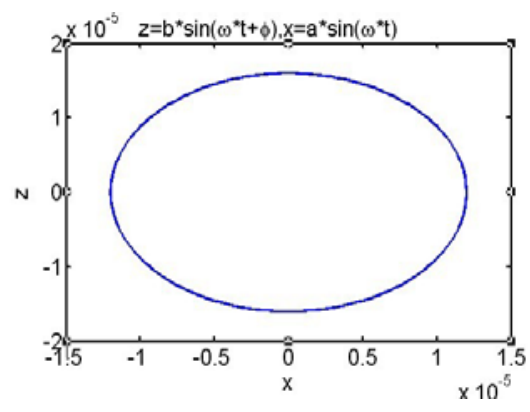

Fig. 5. X, Z composition motion

Fig. 5 is $\mathrm{X}$-axis and Z-axis composition motion, $a$ is major axis and $b$ is the short axis. When the tool does elliptical motion in the $\mathrm{XZ}$ plane, it does uniform motion in a straight line or the feed motion at the same time with velocity $v=20 \mathrm{~m} / \mathrm{min}$ along the Z-axis. Simulative trajectory is shown in Fig.6. This track is similar to spiral grinding motion trace which is shown by Fig.3(b). 


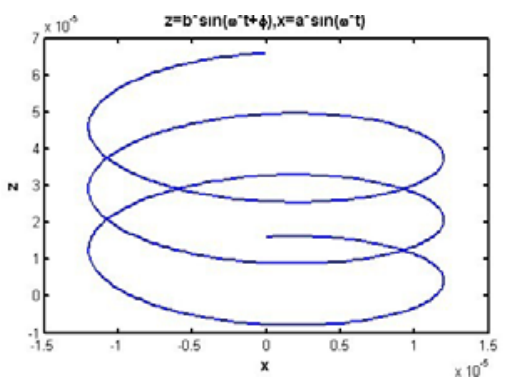

Fig. 6. Synthesis of complex movement

The motion trail is synthesised when $\varphi=0, \varphi=\pi$. According to formula (4) and formula (5), simulative trajectory is shown by Fig.7 and Fig.8.

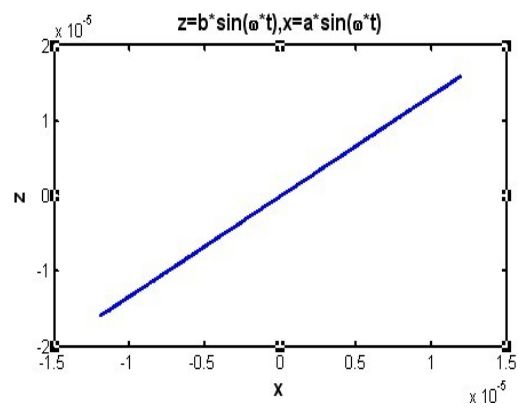

Fig. 7. Motion synthesis $(\varphi=0)$

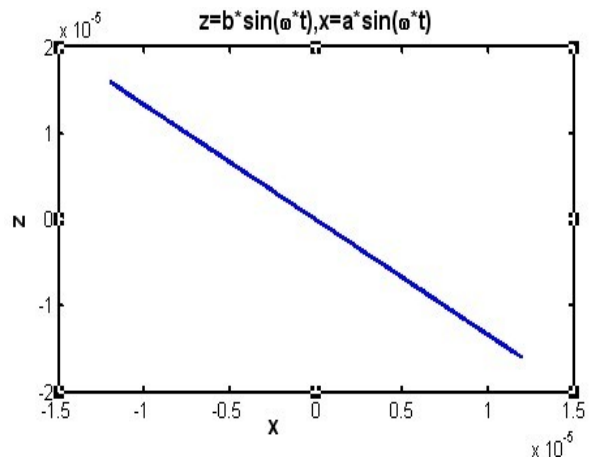

Fig. 8. Motion synthesis $(\varphi=\pi)$

The tool does linear motion in the XZ plane. With the velocity $v=0.6 \mathrm{~m} / \mathrm{min}$, tool along the Z-axis does uniform linear motion, which is also called the feed motion. Simulative trajectory is gotten and shown in Fig.9 and Fig.10. Fig.11 is motion 
synthesis of $\varphi=0$, and $\varphi=\pi$. This track is similar to honing motiton trace which is shown in Fig.3(c).

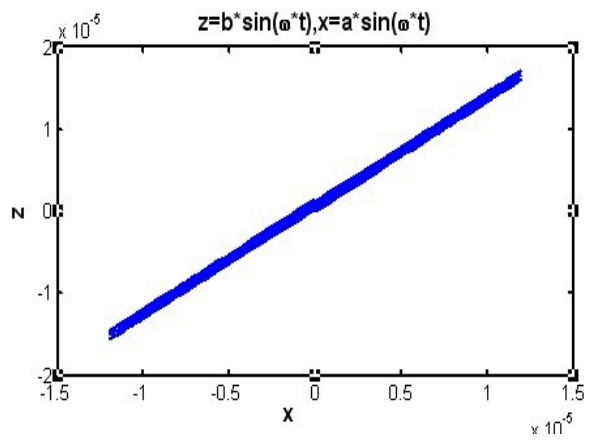

Fig. 9. Synthesis of complex movement $(\varphi=0)$

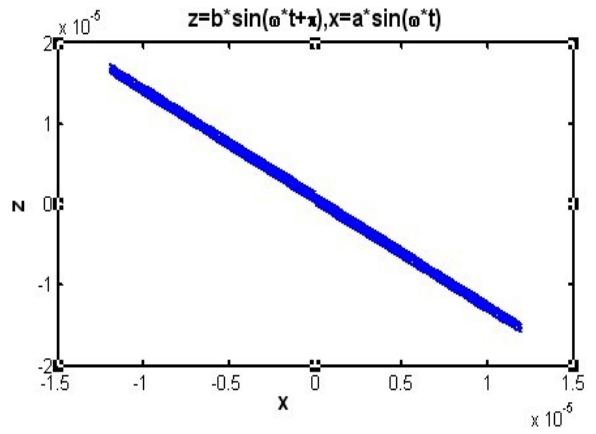

Fig. 10. Synthesis of complex movement $(\varphi=\pi)$

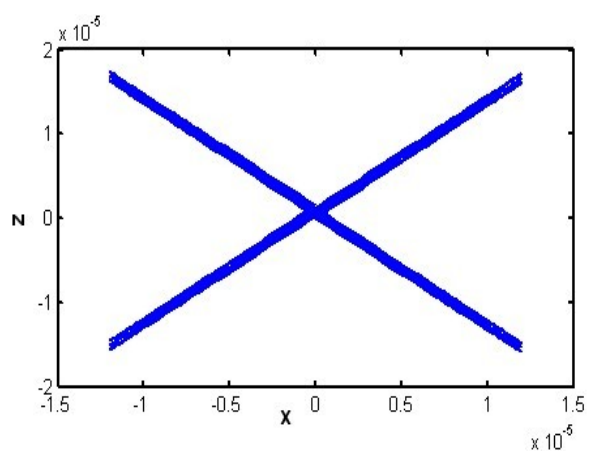

Fig. 11. Synthesis of complex movement $(\varphi=0$ and $\varphi=\pi)$ 


\section{Two-Dimensional Ultrasonic Vibration Turning System Design}

In this paper, ultrasonic transducer uses sandwich piezoelectric ceramic transducer, which is proposed by the French physicist Langevin. This transducer can reduce the problems which are caused by firing and polarization, while reducing the frequency of the piezoelectric ceramic transducer [8].

Fig.12 is diagram of two-dimensional sandwich transducer, which consists of front shroud, back shroud, and piezoelectric ceramic. A stress bolt connects these three parts closely.

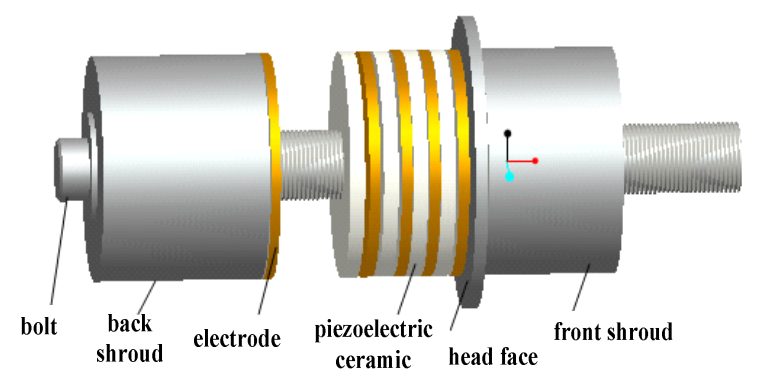

Fig. 12. Diagram of two-dimensional sandwich transducer

Transducer is symmetrical structure, the resonant frequency is $20 \mathrm{KHz}$, and back shroud's material is $45 \#$ steel, outside diameter is $\Phi 55 \mathrm{~mm}$, inside diameter is $\Phi 17 \mathrm{~mm},\left(\rho_{1}=7.8 \mathrm{~g} / \mathrm{cm}^{3}, C_{1}=5.2 \times 10^{5} \mathrm{~cm} / \mathrm{s}, E_{1}=200 \mathrm{GPa}\right)$; The total of piezoelectric ceramics is 5 and its materials use PZT-8, outside diameter is $\Phi 55 \mathrm{~mm}$, inside diameter is $\Phi 17 \mathrm{~mm}$ and thickness is $5 \mathrm{~mm},\left(\rho_{2}=7.5 \mathrm{~g} / \mathrm{cm}^{3}, C_{2}=3.1 \times 10^{5} \mathrm{~cm} / \mathrm{s}, E_{2}=113 \mathrm{GPa}\right)$. Front shroud's material is aluminum, outer diameter is $\Phi 55 \mathrm{~mm}$ and inside diameter is $\Phi 17 \mathrm{~mm},\left(\rho_{3}=2.7 \mathrm{~g} / \mathrm{cm}^{3}, C_{3}=5.1 \times 10^{5} \mathrm{~cm} / \mathrm{s}, E_{3}=75 \mathrm{GPa}\right) . \rho$ is materials density, $C$ is sound velocity, $E$ is elastic modulus. The total of copper electrode is 5 and its thickness is $0.4 \mathrm{~mm}$. The length of back shroud is $l_{1}$ and the length of front shroud is $l_{3}$.

$$
l_{1}=l_{3}=27.3 \mathrm{~mm}
$$

Composite conical horn is shown in Fig.13, which is made of aluminum, outside diameter is $\mathrm{D}_{4}=55 \mathrm{~mm}$, inside diameter is $\mathrm{D}_{5}=17 \mathrm{~mm}, \quad\left(\rho_{4}=\rho_{5}=2.7 \mathrm{~g} / \mathrm{cm}^{3}\right.$, $\left.C_{4}=C_{5}=5.1 \times 10^{5} \mathrm{~cm} / \mathrm{s}, E_{4}=E_{5}=75 \mathrm{GPa}\right)$. 


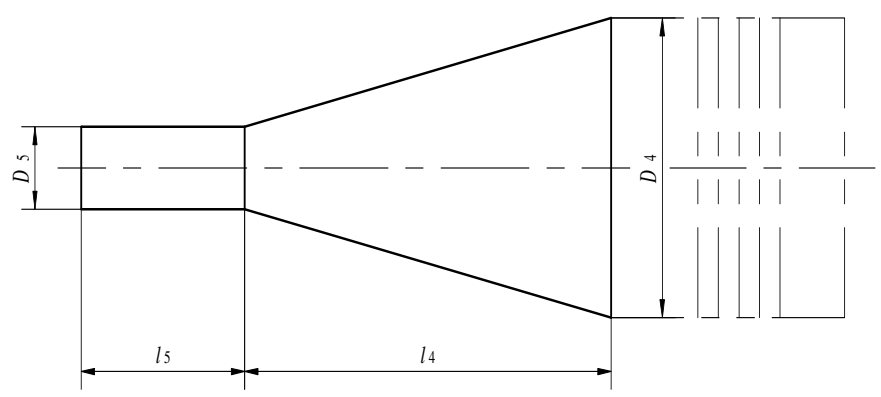

Fig. 13. Diagram of composite conical horn

Frequency equation:

$$
\begin{aligned}
& \tan k l_{5}=\frac{N \alpha}{k}-\tan \left(k l_{4}+\alpha_{2}\right) \\
& \tan \alpha_{2}=\frac{\alpha}{k}, \quad \alpha=\frac{N-1}{N} \times \frac{1}{l_{4}}, N=\frac{D_{4}}{D_{5}} \quad k=\frac{\omega}{C} ;
\end{aligned}
$$

Displacement node $x_{0}$ :

$$
k x_{0}=\left\{\begin{array}{l}
\frac{\pi}{2}-\alpha_{2}, k l_{5}<\frac{\pi}{2} \\
k l_{4}+\left(k l_{5}-\frac{\pi}{2}\right), k l_{5}>\frac{\pi}{2}
\end{array}\right.
$$

Amplification factor $M$ :

$$
M=\left|N\left(\cos k l_{4}-\frac{\alpha}{k} \sin k l_{4}\right) \frac{1}{\cos k l_{5}}\right|
$$

$N$ is area factor and $k$ is wave number. According to formula (7) and formula (9), the simulative curve is shown in Fig.14.

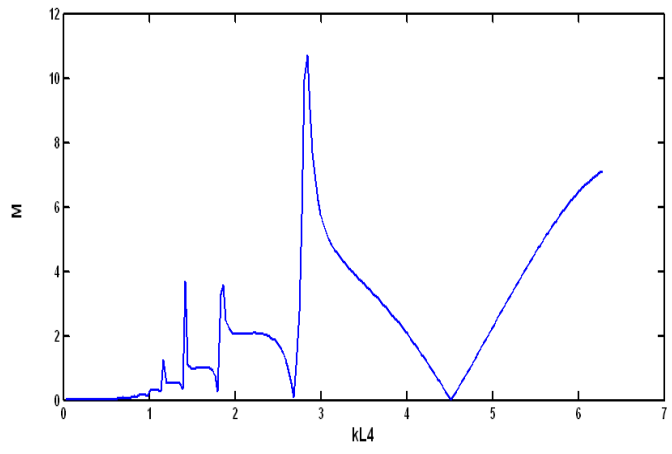

Fig. 14. The curve about $k l_{4}$ and $M$ 


$$
k l_{4}=2.8416, M_{\max }=10.72, l_{4}=0.115 \mathrm{~m}, k l_{5}=1.275, l_{5}=0.052 \mathrm{~m},
$$
$k l_{5}<\pi / 2, k x_{0}=\pi / 2-\alpha_{2}, x_{0}=0.052 \mathrm{~m}$. Nodal plane places $l_{5}=0.052 \mathrm{~m}$ from the big end of the cone.

\section{Two-Dimensional Ultrasonic Vibration System Dynamics Simulation}

Piezoelectric ceramic uses Solid5 in ANSYS element library to establish solid model [9]. Solid5 is used in thermal, magnetic, acoustic, piezoelectric and analysis of three-dimensional coupled field. This unit has eight nodes, and each node has six degrees of freedom. As for the piezoelectric coupling analysis, Solid5 has large deformability.

For others used Solid95. Its unit has 20 nodes and each unit also has X,Y,Z freedom. Solid95 is higher than Solid45, and it doesn't reduce the accuracy of the case.

Piezoelectric ceramic disks are made of PZT-8, and they are thickness polarization. The polarized piezoelectric ceramic is anisotropic, so it is different from isotropic materials. The dielectric constant matrix, the piezoelectric constant matrix and elasticity matrix are given as follows.

Dielectric constant matrix $[\varepsilon] F / m$ :

$$
[\varepsilon]=\left[\begin{array}{ccc}
904 & 0 & 0 \\
0 & 904 & 0 \\
0 & 0 & 576
\end{array}\right]
$$

Piezoelectric constant matrix $[e] \mathrm{c} / \mathrm{m}^{3}$ :

$$
[e]=\left[\begin{array}{ccc}
0 & 0 & -4 \\
0 & 0 & -4 \\
0 & 0 & 13.4 \\
0 & 0 & 0 \\
0 & 10.3 & 0 \\
10.3 & 0 & 0
\end{array}\right]
$$




$$
\begin{gathered}
\text { Elasticity matrix }[C] N / \mathrm{m}^{2} \text { : } \\
{[C]=\left[\begin{array}{cccccc}
13.7 & 6.8 & 7.3 & 0 & 0 & 0 \\
0 & 13.7 & 7.3 & 0 & 0 & 0 \\
0 & 0 & 12.1 & 0 & 0 & 0 \\
0 & 0 & 0 & 3.1 & 0 & 0 \\
0 & 0 & 0 & 0 & 3.1 & 0 \\
0 & 0 & 0 & 0 & 0 & 3.4
\end{array}\right] \times 10^{10}}
\end{gathered}
$$

The horn is a axial symmetry revolving body, and uses bottom-up model. The points generate lines, and lines generate a axial plane. The model of the two-dimensional ultrasonic vibration system is obtained by the axial plane rotated $360^{\circ}$ around the axis. Free mesh is used to generate finite element mesh, which is shown in Fig.15.

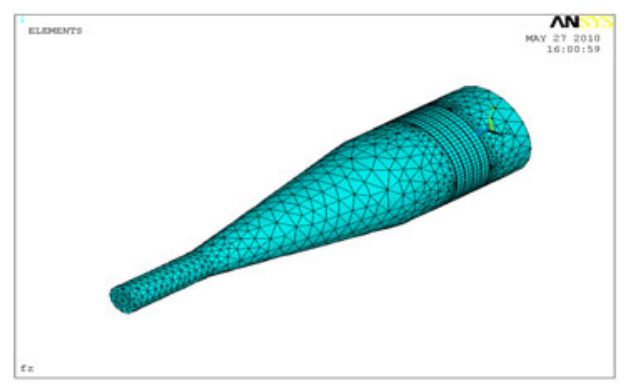

Fig. 15. Finite element mesh

Mode extraction method is subspace iteration method, and the modal number is 10 . In modal analysis, side surface of all nodes between piezoelectric ceramic and front shroud constrain Y displacement, as shown in Fig.16. The electrode voltage $(V=0)$ is coupled on upper and lower surface, which is shown in Fig.17.

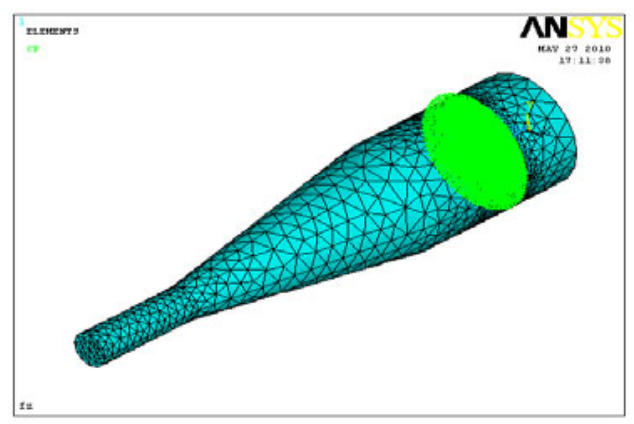

Fig. 16. Constrained Y displacement 


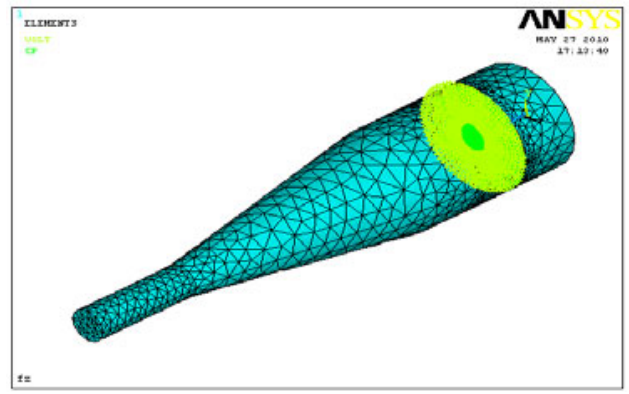

Fig. 17. Coupled the electrode voltage

Through the modal operation, the free resonance frequency is $22255 \mathrm{~Hz}$ in the short-circuit state. This is close to the theoretical calculation of $20 \mathrm{kHz}$. By modal expansion analysis, The relative displacement nephogram is shown in Fig.18.

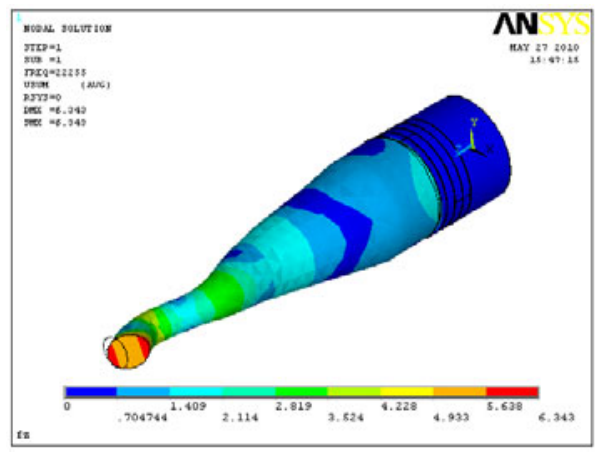

Fig. 18. Relative displacement nephogram

\section{Conclusion}

Traditional ultrasonic vibration turning mechanism and the pros and cons of the processing are analysed in this paper. On this basis, the imitating grinding of twodimensional ultrasonic vibration turning is proposed. The structure of two-dimensional ultrasonic vibration transducer is different from the structure of traditional longitudinal ultrasonic transducer. Necessary grinding track and honing track can be obtained by changing the voltages that apply to piezoelectric ceramic. Meanwhile, the design methods of transducer and horn are given, and which realize the transducer and horn modal analysis by using finite element software. The research improves the machining precision, and makes the application of this technology possibly in ultraprecision machining. 
Acknowledgement. The authors gratefully acknowledge the financial support provided by the University-Industry Cooperation Project of Jiangxi Education Department (Grant No. GJJ09005).

\section{References}

1. Cao, F.: Ultrasonic Machining Technology. Chemical Industry Press, Beijing (2005)

2. Yuan, J., Li, X., Chang, D.: Press Ultrasonic Elliptical Vibration Cutting. Aeronautical Manufacturing Technology 4, 92-93 (2005)

3. Shaomoto, E., Moriwaki, T.: Study on Elliptical Vibration Cutting. Manufacturing Technology 43(1), 35-36 (1994)

4. Lu, Z., Yang, L.: Analysis and Simulation of Mechanism in Ultrasonic-Vibration Cutting Based on Dynamic Fracture Mechanics. Journal of Harbin Institute of Technology 40(9), 1400-1401 (2008)

5. Wu, H., Cao, L., Liu, J.: Analysis of Kinematic Geometry on Face Grinding Process on Lapping Machines. Mechanical Engineering 38(6), 144-146 (2002)

6. Chang, Q., Liu, Y., Ye, S.: Computer Simulation Technology for Precision Lapping Process of Workpiece. Computer Simulation 24(5), 249-250 (2007)

7. Tian, Y.B., Zhou, L., Shimizu, J.: Elimination of Surface Scratch/Texture on The Surface of Single Crystal Si Substrate in Chemo-mechanical Grinding (cmg) Process. Applied Surface Science 255(7), 4205-4211 (2009)

8. Lin, S.: Ultrasonic Transducer Principle and Design. Science Press, Beijing (2004)

9. Qi, J., Li, Z., Zhao, C., Huang, W.: Linear Ultrasonic Motor with Two Degree of Freedom Using Longitudinal and Bending Vibration Modes. Maxon Motor. 12, 18-19 (2005) 